\title{
Boron-Based Organometallic Nanostructures: Hydrogen Storage Properties and Structure Stability
}

\author{
Yufeng Zhao, ${ }^{\star,} \dagger$ Mark T. Lusk, ${ }^{\ddagger}$ Anne C. Dillon, ${ }^{\dagger}$ Michael J. Heben, ${ }^{\dagger}$ and \\ Shengbai B. Zhang ${ }^{\dagger}$ \\ National Renewable Energy Laboratory, Golden, Colorado 80401, and \\ Colorado School of Mines, Colorado 80401
}

Received September 10, 2007; Revised Manuscript Received November 12, 2007

\begin{abstract}
Transition-metal (TM) boride and carboride nanostructures are studied as model organometallic materials for hydrogen storage. The dispersed TM atoms function as $\mathrm{H}_{2}$ sorption centers on the surface of the boron or carbon-boron substrate. The flexibility offered in the variety of possible structures permits the study of the effect of the TM-TM distance on the storage capacity. When the TMs are too close to one another, TM-TM bonding reduces the capacity. Even when separated by distances larger than the normal TM-TM bond length, delocalization of TM valence electrons can still lower the hydrogen capacity. An optimal TM-TM distance for the structural motifs studied here is $\sim 6 \AA$. Our study also permitted the evaluation of new TM boride nanostructures. We predict a low-energy single-walled scandium triboride $\left(\mathrm{ScB}_{3}\right)$ nanotube that can bind $\sim 6.1$ wt $\%$ hydrogen with a binding energy of $22 \sim 26 \mathrm{~kJ} / \mathrm{mol}$.
\end{abstract}

Introduction. Recent theoretical demonstrations of highcapacity, ambient-temperature, reversible hydrogen sorption using transition metals (TMs) complexed to carbon frameworks $^{1,2}$ have prompted many new designs for hydrogen storage materials (HSMs). ${ }^{3-12}$ Experimental investigations of such complexes have already been undertaken, ${ }^{13,14}$ and substantial enhancement of dihydrogen binding has been shown in reduced microporous titanium oxides and Ti-doped silica. ${ }^{15,16}$

The original proposals predicted high reversible capacities for isolated TM atoms supported on carbon superstructures. The stability of the arrangements have been considered a weakness in the design, ${ }^{17}$ but molecular dynamics simulations with boron substitution indicated that structural integrity would be maintained to at least $1000 \mathrm{~K} .{ }^{1}$ A related $\mathrm{Li}_{12} \mathrm{C}_{60}$ cluster arrangement was proposed to be more stable; ${ }^{18}$ unfortunately, the binding energy of $\mathrm{H}_{2}$ to $\mathrm{Li}_{12} \mathrm{C}_{60}$ is too low for practical application, and the $\mathrm{Li}$ atoms will likely cluster once the $\mathrm{Li}_{12} \mathrm{C}_{60}$ forms within a bulk setting. ${ }^{19,20}$

Stimulated by the idea that boron atoms in the substrate may stabilize the dispersed array of TM atoms, ${ }^{1}$ Meng et al. recently proposed a metal-diboride nanotube ${ }^{21}$ as a means to lock the TM atoms in place. In this case, charge transfer from the TM atoms to the boron atoms causes the formation of a layered honeycomb network analogous to graphene. Boron can also form a great variety of organometallic

\footnotetext{
* Corresponding author. E-mail: yufeng_zhao@nrel.gov.

National Renewable Energy Laboratory.

$\doteqdot$ Department of Physics, Colorado School of Mines.
}

structures with TMs and carbon atoms, such as the so-called metallacarboranes ${ }^{22}$ and metal carborides, ${ }^{23}$ so it is interesting to consider other ways of complexing TMs with boron and carbon.

Because dispersed TM atoms in organometallic HSMs function as hydrogen sorption centers, it is natural to consider how the density of TM atoms on the substrate affects the hydrogen storage capacity. For example, one may ask: Does the hydrogen capacity increase as the TM density increases? Interestingly, the TM-diboride nanostructures ${ }^{21,24-27}$ with a relatively high TM density do not provide a higher hydrogen capacity ${ }^{21}$ as compared to the organometallic buckyballs. ${ }^{1}$ This suggests that a closer investigation of the relationship between TM density and hydrogen capacity is warranted.

In this study, we show that $\mathrm{TM}$ atoms, $\mathrm{Sc}$ and $\mathrm{Ti}$, can bind externally to fullerene-like cages of the general formula $\mathrm{C}_{x} \mathrm{~B}_{60-x}(x=0,36)$. Because the TM-TM distance is tuned by varying $x$, we can investigate how the hydrogen capacity depends on the surface density of TM atoms. We also show that the metal-coated boron cages are metastable, whereas a type of Sc-embedded $\mathrm{ScB}_{3}$ nanotube has a much lower energy and good properties for hydrogen storage. Furthermore, following analogies to nanostructures that are known to exist, we suggest that some of these new nanomaterials may be readily synthesized.

Theory and Computational Details. The gravimetric capacity, $C_{\mathrm{W}}$, of an HSM is related to the number of sorption centers per unit HSM mass, $D_{\mathrm{s}}$, through the number of $\mathrm{H}$ 
species ( $n_{\mathrm{H}}$, in either atomic or molecular forms) bound to a sorption center and the atomic mass of the hydrogen atom, $m_{\mathrm{H}}$, as the following:

$$
C_{\mathrm{W}}=D_{\mathrm{s}} n_{\mathrm{H}} m_{\mathrm{H}}
$$

Focusing on organometallic HSMs with TM atoms acting as sorption centers, we consider the 18-electron rule where the number of $\mathrm{H}$ species bound to each TM atom is

$$
n_{\mathrm{H}}=18-n_{\mathrm{v}}^{\mathrm{e}}-n_{\mathrm{s}}^{\mathrm{e}}-n_{\mathrm{m}}^{\mathrm{e}}
$$

where the number of valence electrons in the free TM atom, $n_{\mathrm{v}}^{\mathrm{e}}$, is a constant once the TM is chosen, and $n_{\mathrm{s}}^{\mathrm{e}}$ and $n_{\mathrm{m}}^{\mathrm{e}}$ are the number of electrons contributed to the TM orbitals by, respectively, the substrate and neighboring TM atoms. When the TM atoms are bound to the substrate in a stable configuration, $n_{\mathrm{s}}^{\mathrm{e}}$ is also a constant. However, $n_{\mathrm{m}}^{\mathrm{e}}$ is affected by the TM-TM distance and hence by the TM density $D_{\text {s }}$. When the TM atoms are far away from one another, $n_{\mathrm{m}}^{\mathrm{e}}$ can be neglected and $C_{\mathrm{W}}$ increases with increasing $D_{\mathrm{s}}$. When TMs are close to one another, neighboring TMs interact and $n_{\mathrm{m}}^{\mathrm{e}}$ is the number of TM-TM bonds. Obviously, TM separation is desired to avoid a situation in which the TM orbitals are involved in forming TM-TM bonds such that $n_{\mathrm{H}}$ is small. However, it is unclear how the TM-TM interaction affects hydrogen capacity in the intermediate range of TM-TM distances, which is longer than their normal bonds but shorter than that of complete separation.

An investigation of the TM density requires changing the ratio of the TM atoms to the substrate atoms. If we assume that each boron atom requires one electron to form a carbonlike electronic shell, the metal-boron stoichiometric ratio can take on several different values spanning from the 1:2 ratio investigated by Meng et al. ${ }^{21}$ to $1: 3$ or $1: 4$ due to the different TM valences. For example, $\mathrm{ScB}_{3}$ and $\mathrm{TiB}_{4}$ could be stable in nanoparticles in which the TM density is lowered. The TM density could be further lowered if carbon atoms are introduced. These considerations lead to several new nanostructured HSMs.

We used the spin-polarized first-principles density functional theory method implemented in the Vienna ab initio simulation package. ${ }^{28} \mathrm{~A}$ plane-wave basis set ( $400 \mathrm{eV}$ cutoff) was used in combination with an all-electronlike projectoraugmented wave potential and Perdew-Burke-Ernserhof exchange correlation functional within the generalized gradient approximation. ${ }^{29}$ The periodic, cubic unit-cell is given a dimension of $25 \AA$ to maintain sufficient vacuum around the nanoparticles. For the one-dimensional nanotubes, the axial period is optimized in the total-energy calculation. The optimized structures are considered as equilibrium when the maximum force acting on the atoms decreases below 0.03 $\mathrm{eV} / \mathrm{A}$. The definition of the binding energy of hydrogen can be found in refs 1 and 9 .

Results and Discussion. We first calculate the metaltriboride nanocages $\mathrm{Sc}_{20} \mathrm{~B}_{60}$ and $\mathrm{Ti}_{20} \mathrm{~B}_{60}$ with each $\mathrm{TM}$ (Sc or Ti) atom sitting on top of the center of a hexagonal ring.
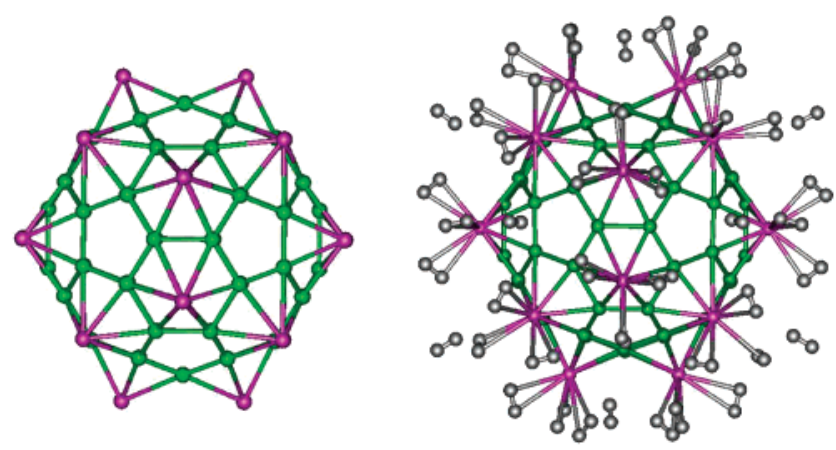

Figure 1. A metal-boride buckyball $\mathrm{Sc}_{20} \mathrm{~B}_{60}$ (left) adsorbs $72 \mathrm{H}_{2}$ or 8.6 wt \% (right). Each $\mathrm{Sc}$ atom binds three $\mathrm{H}_{2}$, and each pentagon ring binds one $\mathrm{H}_{2}$. Green and pink balls are $\mathrm{B}$ and $\mathrm{Sc}$ atoms, respectively.

Table 1. HOMO-LUMO Gaps for the Nanoparticles before and after Hydrogen Charging

\begin{tabular}{lclc}
\hline \multicolumn{1}{c}{ structure } & gap $(\mathrm{eV})$ & \multicolumn{1}{c}{ structure } & gap $(\mathrm{eV})$ \\
\hline $\mathrm{Ti}_{20} \mathrm{~B}_{60}$ & 0.02 & $\mathrm{Sc}_{20} \mathrm{~B}_{60}$ & 0.20 \\
$\mathrm{H}_{104} \mathrm{Ti}_{20} \mathrm{~B}_{60}$ & 0.28 & $\mathrm{H}_{144} \mathrm{Sc}_{20} \mathrm{~B}_{60}$ & 0.27 \\
$\mathrm{Ti}_{12} \mathrm{~B}_{24} \mathrm{C}_{36}$ & 0.06 & $\mathrm{Sc}_{12} \mathrm{~B}_{24} \mathrm{C}_{36}$ & 0.12 \\
$\mathrm{H}_{132} \mathrm{Ti}_{12} \mathrm{~B}_{24} \mathrm{C}_{36}$ & 1.43 & $\mathrm{H}_{144} \mathrm{Sc}_{12} \mathrm{~B}_{24} \mathrm{C}_{36}$ & 1.48
\end{tabular}

Table 2. Hydrogen Storage Property of Metal-Boride and Metal-Carboride Nanostructures

\begin{tabular}{lccc}
\hline \multicolumn{1}{c}{ structure } & $\begin{array}{c}\mathrm{TM}-\mathrm{TM} \\
\text { distance }(\AA)\end{array}$ & $\begin{array}{c}\mathrm{H}_{2} \text { binding } \\
(\mathrm{kJ} / \mathrm{mol})\end{array}$ & $\begin{array}{r}\text { capacity } \\
(\text { wt \%) }\end{array}$ \\
\hline $\mathrm{TiB}_{2}$ tube $^{a}$ & $3.0-3.3$ & $19-58$ & 5.5 \\
$\mathrm{TiB}_{3}\left(\mathrm{Ti}_{20} \mathrm{~B}_{60}\right)$ & 3.69 & $12-36$ & 6.5 \\
$\mathrm{TiB}_{2} \mathrm{C}_{3}\left(\mathrm{Ti}_{12} \mathrm{~B}_{24} \mathrm{C}_{36}\right)$ & 5.47 & 38 & 8.6 \\
$\mathrm{ScB}_{3}\left(\mathrm{Sc}_{20} \mathrm{~B}_{60}\right)$ & 3.84 & $15-25$ & 8.6 \\
$\mathrm{ScB}_{2} \mathrm{C}_{3}\left(\mathrm{Sc}_{12} \mathrm{~B}_{24} \mathrm{C}_{36}\right)$ & 5.62 & 39 & 10.5 \\
${ }^{a}$ Taken from ref 21. & & &
\end{tabular}

Notice that if the TM atoms are replaced with B atoms, these structures change into the recently predicted $\mathrm{B}_{80}$ fullerene. ${ }^{30}$ Note that $\mathrm{Sc}_{20} \mathrm{~B}_{60}$ has exactly the same $I_{\mathrm{h}}$ symmetry and the same number of valence electrons as $\mathrm{B}_{80}$. Figure 1 (left) shows the optimized structure of the $\mathrm{Sc}_{20} \mathrm{~B}_{60}$ metal-triboride buckyball. In this case, each $\mathrm{Sc}$ atom can bind three $\mathrm{H}_{2}$ ligands (Figure 1, right) with a binding energy of $25.1 \mathrm{~kJ} /$ mol $\mathrm{H}_{2}$. In addition to the $\mathrm{H}_{2}$ molecules bound to the $\mathrm{Sc}$ atoms, one extra $\mathrm{H}_{2}$ can bind on top of a pentagonal ring with a binding energy of $15.4 \mathrm{~kJ} / \mathrm{mol} \mathrm{H}_{2}$. In $\mathrm{Ti}_{20} \mathrm{~B}_{60}$, each Ti atom binds two $\mathrm{H}_{2}\left(35.6 \mathrm{~kJ} / \mathrm{mol} \mathrm{H}_{2}\right)$, and one more $\mathrm{H}_{2}$ is bound $\left(11.6 \mathrm{~kJ} / \mathrm{mol} \mathrm{H}_{2}\right)$ to each pentagon. The highest occupied molecular orbital-lowest unoccupied molecular orbital (HOMO-LUMO) gap of $\mathrm{Sc}_{20} \mathrm{~B}_{60}$ is 10 times larger than that of $\mathrm{Ti}_{20} \mathrm{~B}_{60}$ (Table 1), indicating that the electroncounting rule requires roughly one electron being transferred to each $\mathrm{B}$ atom from the metal atoms.

Although the density of the TM atoms is lower than in $\mathrm{TiB}_{2}$ nanotubes, ${ }^{21}$ these nanostructures store more hydrogen (see Table 2). Notice that the TM-TM distance $(\sim 3.8 \AA)$ in $\mathrm{Sc}_{20} \mathrm{~B}_{60}$ and $\mathrm{Ti}_{20} \mathrm{~B}_{60}$ is larger than that in the $\mathrm{TiB}_{2}$ nanotubes (3.0-3.3 $\AA$ ). The question then arises: Can the hydrogen capacity be increased if the TM-TM distance is 

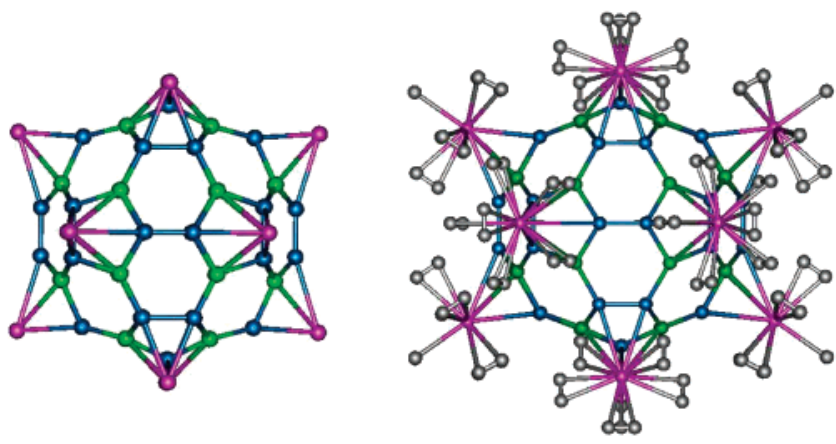

Figure 2. A metal-carboride buckyball $\mathrm{Sc}_{12} \mathrm{~B}_{24} \mathrm{C}_{36}$ (left) adsorbs $72 \mathrm{H}_{2}$ or 10.5 wt \% (right). Each Sc atom binds six $\mathrm{H}_{2}$. Green, blue, and pink balls are B, C, and Sc atoms, respectively.

further enlarged? The TM-TM distance might be increased by simply removing TM atoms, but this would lead to destabilization of the boron cage because boron atoms prefer a high coordination number when there is not sufficient electron donation from metal atoms. Another approach is to replace some of the boron atoms with carbon. This approach appears to be viable because the energetic and thermodynamic favorability of metal carborides has been shown in experimental work. ${ }^{22,23}$

Figure 2 shows a metal-carboride buckyball, $\mathrm{Sc}_{12} \mathrm{~B}_{24} \mathrm{C}_{36}$, in which each $\mathrm{Sc}$ atom covers a pentagonal ring with three $\mathrm{C}$ atoms and two $\mathrm{B}$ atoms. This can be considered a heavily B-doped $\mathrm{C}_{60}$ with Sc decoration. In $\mathrm{Sc}_{12} \mathrm{~B}_{24} \mathrm{C}_{36}$, each Sc atom binds as many as six $\mathrm{H}_{2}$ with an average binding energy of $38.6 \mathrm{~kJ} / \mathrm{mol} \mathrm{H}_{2}$. We also calculated the structural analog for $\mathrm{Ti}$, that is, $\mathrm{Ti}_{12} \mathrm{~B}_{24} \mathrm{C}_{36}$, and found that each $\mathrm{Ti}$ atom can bind one hydride and five $\mathrm{H}_{2}$. The binding energy of the hydride $\left(179 \mathrm{~kJ} / \mathrm{mol} \mathrm{H}_{2}\right)$ is too high for the $\mathrm{H}$ atoms to be retrieved at ambient conditions but that of the dihydrogen $(37.6 \mathrm{~kJ} /$ mol $\left.\mathrm{H}_{2}\right)$ is nearly ideal. Compared with the $\mathrm{M}_{20} \mathrm{~B}_{60}(\mathrm{M}=$ $\mathrm{Sc}, \mathrm{Ti}$ ) case, the hydrogen capacity in $\mathrm{M}_{12} \mathrm{~B}_{24} \mathrm{C}_{36}$ is increased though the metal density is decreased.

These results indicate that the distance between TM centers is a key factor in the design of optimal organometallic HSMs. Table 2 summarizes the relationship between the TM-TM distances and the capacity within the structural motifs investigated here. It is seen that the hydrogen capacity increases with TM-TM distance. However, this will not be a monotonic trend in the limit, because a low density of metal atoms will eventually reduce the hydrogen uptake per unit mass of HSM, as expected from eq 1.

One might conjecture that the relatively low capacity at high metal density is mainly due to steric effects associated with the metal species being too close. ${ }^{21}$ However, this cannot explain why $\mathrm{Ti}_{20} \mathrm{~B}_{60}$ stores less hydrogen than $\mathrm{Sc}_{20} \mathrm{~B}_{60}$ where the metal-metal separation is similar. We found that the degree of electron localization plays a key role. Even at a TM-TM distance much longer than their direct bonds, the TM atoms tend to interact with each other through valenceelectron delocalization mediated by the substrate. Because Ti has one more valence electron than $\mathrm{Sc}$, the delocalization effect in $\mathrm{Ti}_{20} \mathrm{~B}_{60}$ is more pronounced than in $\mathrm{Sc}_{20} \mathrm{~B}_{60}$, and this is reflected in the gaps listed in Table 1. Figure 3 shows
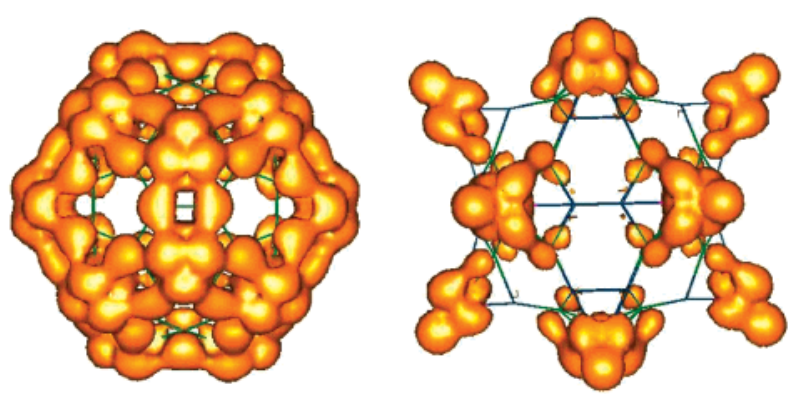

Figure 3. Charge density of the highest occupied band for $\mathrm{Ti}_{20} \mathrm{~B}_{60}$ (left) and $\mathrm{Ti}_{12} \mathrm{~B}_{24} \mathrm{C}_{36}$ (right) nanoparticles.

the highly delocalized electronic states in the highest occupied band of $\mathrm{Ti}_{20} \mathrm{~B}_{60}$. TM valence electrons are delocalized in both $\mathrm{Sc}_{20} \mathrm{~B}_{60}$ and $\mathrm{Ti}_{20} \mathrm{~B}_{60}$ due to an intermediate $\mathrm{TM}-\mathrm{TM}$ distance of 3.7 3.8 $\AA$. In contrast, as the TMTM distance increases further to $5.5 \sim 5.6 \AA$ in $\mathrm{Ti}_{12} \mathrm{~B}_{24} \mathrm{C}_{36}$ and $\mathrm{Sc}_{12} \mathrm{~B}_{24} \mathrm{C}_{36}$, the TM valence electrons are highly localized around the pentagonal rings (Figure 3). Such an electronic effect is also reflected in the chemistry of the nanoparticles. As shown in Table 1, the fully charged $\mathrm{Ti}_{20} \mathrm{~B}_{60}$ and $\mathrm{Sc}_{20} \mathrm{~B}_{60}$ species (i.e., $\mathrm{H}_{104} \mathrm{Ti}_{20} \mathrm{~B}_{60}$ and $\mathrm{H}_{144} \mathrm{Sc}_{20} \mathrm{~B}_{60}$ ) have a much narrower gap than the fully charged $\mathrm{Sc}_{12} \mathrm{~B}_{24} \mathrm{C}_{36}$ and $\mathrm{Ti}_{12} \mathrm{~B}_{24} \mathrm{C}_{36}$ (i.e., $\mathrm{H}_{132} \mathrm{Ti}_{12} \mathrm{~B}_{24} \mathrm{C}_{36}$ and $\mathrm{H}_{144} \mathrm{Sc}_{12} \mathrm{~B}_{24} \mathrm{C}_{36}$ ), which implies that $\mathrm{H}_{104} \mathrm{Ti}_{20} \mathrm{~B}_{60}$ and $\mathrm{H}_{144} \mathrm{Sc}_{20} \mathrm{~B}_{60}$ are chemically unsaturated. The electron delocalization does not fully saturate the TM orbitals, yet the hydrogen capacity is significantly reduced. Notice that in $\mathrm{H}_{132} \mathrm{Ti}_{12} \mathrm{~B}_{24} \mathrm{C}_{36}$ and $\mathrm{H}_{144} \mathrm{Sc}_{12} \mathrm{~B}_{24} \mathrm{C}_{36}$ the $\mathrm{TM}$ atom, $\mathrm{H}_{2}$ molecules, and $\mathrm{H}$ atoms satisfy the 18-e rule in each pentagonal ring and create local aromaticity. The hydrogen capacity reaches its maximum at a TM-TM distance of $\sim 6$ $\AA$, which should be near the optimized value.

To evaluate the stability of the nanostructures, we calculated the energies of the $\mathrm{Sc}_{20} \mathrm{~B}_{60}$ isomers with some of the Sc atoms moved into the $\mathrm{B}_{60}$ cage. This may be considered as a perturbation to the targeted structure. Figure 4 shows that the total energy drops by $0.58,1.15$, and $6.29 \mathrm{eV}$ with 1,2 , and $4 \mathrm{Sc}$ moved inside, respectively. Extending this type of study to the $\mathrm{TiB}_{2}$ tube, we may expect similar results because TM atoms favor higher coordination numbers. As more TM atoms go inside the tube (or cage), the structure should collapse and form a solid particle. However, there are some hollow structures that are stable when boron is coordinated with five neighbors. For example, we have found an exceptionally stable nanotube of formula $\mathrm{ScB}_{3}$ in which the Sc atoms are embedded in the wall and all the boron atoms have 5-fold coordination, as shown in Figure 5 (lower panel). In this fully relaxed structure with an axial period of $5.90 \AA$, the nearest $\mathrm{B}-\mathrm{B}, \mathrm{B}-\mathrm{Sc}$, and $\mathrm{Sc}-\mathrm{Sc}$ distances are $1.61,2.43$, and $3.48 \AA$, respectively. Our calculation shows that the energy of the $\mathrm{Sc}$-embedded $\mathrm{ScB}_{3}$ nanotube is 0.8 $\mathrm{eV}$ lower per $\mathrm{ScB}_{3}$ unit than the Sc-coated one (Figure 5, upper panel). Compared to the $\mathrm{Sc}_{20} \mathrm{~B}_{60}$ buckyball in Figure 1 and its lowest-energy solid isomer (in Figure 4 with four Sc inside), the Sc-embedded $\mathrm{ScB}_{3}$ nanotube is 0.60 and $0.29 \mathrm{eV}$ lower, respectively, in total energy per $\mathrm{ScB}_{3}$ unit. This implies that a variety of cagelike TM boride nanostructures may exist. 


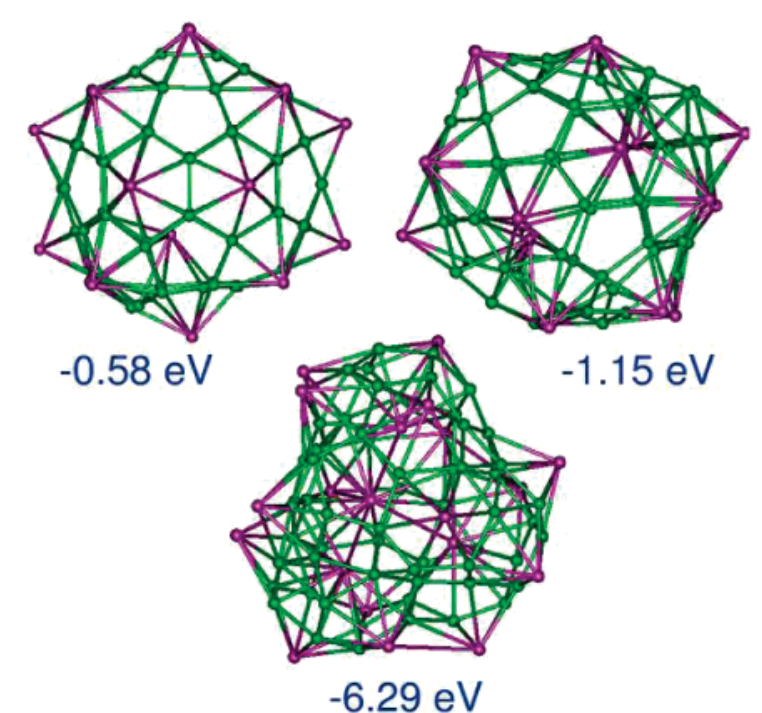

Figure 4. Three $\mathrm{Sc}_{20} \mathrm{~B}_{60}$ isomers with 1,2 , and 4 of the $20 \mathrm{Sc}$ atoms inside the $\mathrm{B}_{60}$. Their total energies are $0.58,1.15$, and 6.29 $\mathrm{eV}$ lower, respectively, than the Sc-coated $\mathrm{B}_{60}$ (as shown in Figure $1)$.

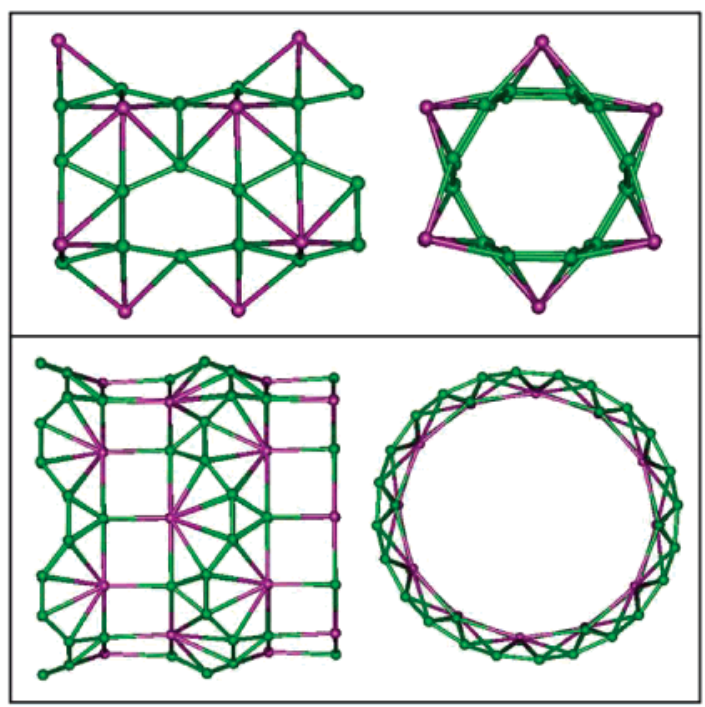

Figure 5. Predicted Sc-coated (upper panel) and Sc-embedded (lower panel) $\mathrm{ScB}_{3}$ nanotubes. Side views and axial views are shown. The total energy of the Sc-embedded nanotube is $0.8 \mathrm{eV}$ lower per stoichiometric unit $\left(\mathrm{ScB}_{3}\right)$ than the Sc-coated one.

To further evaluate the feasibility of synthesis of the Scembedded $\mathrm{ScB}_{3}$ nanotube, we compare here the formation energy of this $\mathrm{ScB}_{3}$ nanotube with the famous $\mathrm{Ti}_{8} \mathrm{C}_{12}$ MetCar molecule, via the following reactions:

$$
\begin{gathered}
\mathrm{ScB}_{3} \leftrightarrow \mathrm{ScB}_{2}+\mathrm{B} \\
\mathrm{Ti}_{8} \mathrm{C}_{12} \leftrightarrow 8 \mathrm{TiC}+4 \mathrm{C}
\end{gathered}
$$

The formulas on the left-hand side are nanostructures, and those on the right-hand side represent bulk materials from which the nanostructures are formed. The well-known scandium diboride $\left(\mathrm{ScB}_{2}\right)$ and the titanium carbide $(\mathrm{TiC})$ are, respectively, hexagonal closed packed HCP-A3 and rock salt
B 1 crystals. Bulk boron is the ground-state $\beta$-boron ( $\mathrm{R}-105)$, and the bulk carbon is graphene. According to our calculation, the formation energy of the predicted $\mathrm{ScB}_{3}$ nanotube is $0.64 \mathrm{eV}$ per atom as in comparison with that of $1.02 \mathrm{eV}$ per atom of $\mathrm{Ti}_{8} \mathrm{C}_{12}$ molecules. Notice that the formation energy of $\mathrm{C}_{60}$ is $\sim 0.4 \mathrm{eV}$ per carbon atom with respect to graphene; ${ }^{31}$ this implies a high possibility of existence of the $\mathrm{ScB}_{3}$ nanotubes. As for the synthesis of $\mathrm{M}_{12} \mathrm{~B}_{24} \mathrm{C}_{36}$, the problem is similar to $\mathrm{B}$ substitution of $\mathrm{C}$ in small hydrocarbon molecules in the presence of metal. ${ }^{22}$ For example, the sandwiched organometallic structures containing $\left[\mathrm{C}_{3} \mathrm{~B}_{2} \mathrm{H}_{5}\right]^{2-}$ carborane $^{22}$ could be precursors for synthesis of TM-coated $\mathrm{B}_{24} \mathrm{C}_{36}$ buckyballs.

We also investigated the interaction of the Sc-embedded $\mathrm{ScB}_{3}$ nanotube with hydrogen. Our calculation shows that each boron atom binds one atomic $\mathrm{H}$, and each $\mathrm{Sc}$ atom binds one dihydrogen molecule through Kubas coordination, resulting in a total hydrogen capacity of $6.1 \mathrm{wt} \%$. Interestingly, the binding energy of the atomic hydrogen $(22 \mathrm{~kJ} /$ mol $\mathrm{H}_{2}$ ) is slightly smaller than that of the dihydrogen, 26 $\mathrm{kJ} / \mathrm{mol} \mathrm{H}_{2}$. This means that both these two types of hydrogen can be reversibly charged/discharged at near ambient condition. The hydrogenation/dehydrogenation of boron atoms may be catalyzed by the Sc atom, as has been shown for MetCars. ${ }^{9}$

Conclusion. In summary, we investigated the effect of TM density on hydrogen storage in organometallic structures. Undercoordinated surface TM atoms can interact through surface delocalization of valence electrons, which can lower the hydrogen capacity. There exists an optimal TM-TM distance $(\sim 6 \AA)$ for the maximum hydrogen capacity in organometallic frameworks with dispersed TM atoms. Finally, stable single-walled $\mathrm{ScB}_{3}$ nanotubes are predicted that show potential for hydrogen storage.

Acknowledgment. This work is supported by the Office of Energy Efficiency and Renewable Energy Hydrogen, Fuel Cell, and Infrastructure Technologies Program of the Department of Energy through the Hydrogen Sorption Center of Excellence under Grant DE-AC36-99GO10337. We thank H.-J. Xiang for helpful suggestions.

\section{References}

(1) Zhao, Y.; Kim, Y.-H.; Dillon, A. C.; Heben, M. J.; Zhang, S. B. Phys. Rev. Lett. 2005, 94, 155504.

(2) Yildirim, T.; Ciraci, S. Phys. Rev. Lett. 2005, 94, 175501.

(3) Gagliardi, L. J. Chem. Theory Comput. 2005, 1, 1172-1175.

(4) Shin, W. H.; Yang, S. H.; Goddard, W. A.; Kang, J. K. Appl. Phys. Lett. 2006, 88, 053111.

(5) Lee, H.; Choi, W. I.; Ihm, J. Phys. Rev. Lett. 2006, 97, 056104

(6) Du, A. J.; Smith, S. C.; Yao, X. D.; Lu, G. Q. J. Phys. Chem. B 2006, 110, 21747-21750.

(7) Weck, P. F.; Kumar, T. J. D.; Kim, E.; Balakrishnan, N. J. Chem. Phys. 2007, 126, 094703-6.

(8) Wu, X. J.; Yang, J. L.; Zeng, X. C. J. Chem. Phys. 2006, 125, 044704

(9) Zhao, Y. F.; Dillon, A. C.; Kim, Y. H.; Heben, M. J.; Zhang, S. B. Chem. Phys. Lett. 2006, 425, 273-277.

(10) Durgun, E.; Ciraci, S.; Zhou, W.; Yildirim, T. Phys. Rev. Lett. 2006 97, 226102.

(11) Park, N.; Hong, S.; Kim, G.; Jhi, S. H. J. Am. Chem. Soc. 2007, 129, 8999-9003

(12) Zhao, Y. F.; Heben, M. J.; Dillon, A. C.; Simpson, L. J.; Blackburn, J. L.; Dorn, H. C.; Zhang, S. B. B. J. Phys. Chem. C 2007, 111, $13275-13279$. 
(13) Borchers, C.; Khomenko, T. I.; Morozova, O. S.; Galakhov, A. V.; Kurmaev, E. Z.; McNaughton, J.; Yabloskikh, M. V.; Moewes, A. J. Phys. Chem. B 2006, 110, 196-204.

(14) Wang, X. L.; Tu, J. P. Appl. Phys. Lett. 2006, 89, 064101.

(15) Hu, X.; Skadtchenko, B. O.; Trudeau, M.; Antonelli, D. M. J. Am. Chem. Soc. 2006, 128, 11740-11741.

(16) Hu, X.; Trudeau, M.; Antonelli, D. M. Chem. Mater. 2007, 19, 1388 1395 .

(17) Sun, O.; Wang, Q.; Jena, P.; Kawazoe, Y. J. Am. Chem. Soc. 2005 , 127, 14582-14583.

(18) Sun, Q.; Jena, P.; Wang, Q.; Marquez, M. J. Am. Chem. Soc. 2006, 128, 9741-9745.

(19) Buhl, M. Z. Anorg. Allg. Chem. 2000, 626, 332-337.

(20) Cristofolini, L.; Facci, P.; Fontana, M. P.; Cicognani, G.; Dianoux, A. J. Phys. Rev. B 2000, 61, 3404-3409.

(21) Meng, S.; Kaxiras, E.; Zhang, Z. Nano Lett. 2007, 7, 663-667.

(22) Grimes, R. N. Chem. Rev. 1992, 92, 251-268.
(23) Kriz, O.; Rheingold, A. L.; Shang, M. Y.; Fehlner, T. P. Inorg. Chem. 1994, 33, 3777-3783

(24) Ivanovskaya, V.; Enjashin, A. N.; Sofronov, A. A.; Makurin, Y. N.; Medvedeva, N. I.; Ivanovskii, A. L. J. Mol. Struct. 2003, 625, 9-16.

(25) Nagamatsu, J.; Nakagawa, N.; Muranaka, T.; Zenitani, Y.; Akimitsu, J. Nature 2001, 410, 63-64.

(26) Quandt, A.; Liu, A. Y.; Boustani, I. Phys. Rev. B 2001, 6412.

(27) Zhang, P. H.; Crespi, V. H. Phys. Rev. Lett. 2002, 89, 056403.

(28) Kresse, G.; Furthmuller, J. Phys. Rev. B 1996, 54, 11169-11186.

(29) Perdew, J. P.; Burke, K.; Ernzerhof, M. Phys. Rev. Lett. 1996, 77, 3865-3868.

(30) Szwacki, N. G.; Sadrzadeh, A.; Yakobson, B. I. Phys. Rev. Lett. 2007, 98, 166804.

(31) Chen, H. S.; Kortan, A. R.; Haddon, R. C.; Kaplan, M. L.; Chen, C. H.; Mujsce, A. M.; Chou, H.; Fleming, D. A. Appl. Phys. Lett. 1991, 59, 2956-2958.

NL072321F 